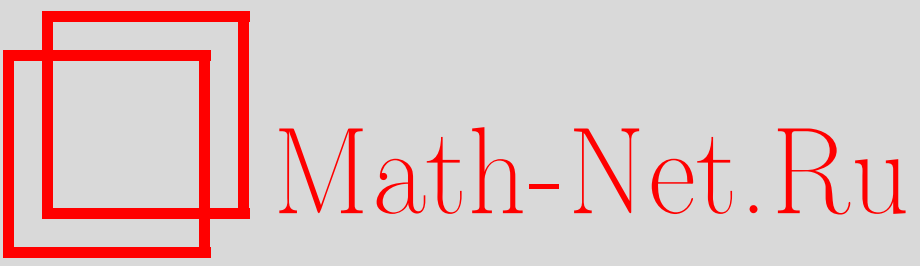

С. М. Натанзон, Топологическая структура пространства голоморфных морфизмов римановых поверхностей, УМН, 1998, том 53, выпуск 2, 155-156

DOI: https://doi.org/10.4213/rm41

Использование Общероссийского математического портала Math-Net.Ru подразумевает, что вы прочитали и согласны с пользовательским соглашением

http://www.mathnet.ru/rus/agreement

Параметры загрузки:

IP : 54.224 .60 .19

26 апреля 2023 г., 14:22:11 


\title{
ТОПОЛОГИЧЕСКАЯ СТРУКТУРА ПРОСТРАНСТВА ГОЛОМОРФНЫХ МОРФИЗМОВ РИМАНОВЫХ ПОВЕРХНОСТЕЙ
}

\author{
C. М. НАТАНЗОН
}

1. Голоморфнымм морфизмом в статье назьвается тройка $(\widetilde{P}, f, P)$, где $\widetilde{P}$ и $P$ - компактные римановы поверхности, а $f: \widetilde{P} \rightarrow P$ - голоморфное разветвленное накрытие. Морфизмы $\left(\widetilde{P}_{1}, f_{1}, P_{1}\right)$ и $\left(\widetilde{P}_{2}, f_{2}, P_{2}\right)$ считаются совпадающими, если существуют биголоморфные отображения $\widetilde{\varphi}: \widetilde{P}_{1} \rightarrow \widetilde{P}_{2}$ и $\varphi: P_{1} \rightarrow P_{2}$ такие, что $\varphi f_{1}=f_{2} \widetilde{\varphi}$.

Морфизмы $\left(\widetilde{P}_{1}, f_{1}, P_{1}\right)$ и $\left(\widetilde{P}_{2}, f_{2}, P_{2}\right)$ называются толологически әквивалентнымии (или имеющими одинаковьй топологический тun), если существуют гомеоморфизмы $\widetilde{\varphi}: \widetilde{P}_{1} \rightarrow \widetilde{P}_{2}$ и $\varphi: P_{1} \rightarrow P_{2}$ такие, что $\varphi f_{1}=f_{2} \widetilde{\varphi}$.

Забиксируем произвольный топологический тип $t$ и рассмотрим множество $H^{t}$ всех морфизмов этого топологического типа. Род $g(t)$ поверхности $P$ и число $n(t)$ критических значений $f$ являются инвариантами топологического типа $t$. В этой заметке мы описьваем естественную топологию в $H^{t}$ и даем набросок доказателшства следующей теоремы.

Теорема. Пусть $2 g(t)+n(t)>2$. Тогда пространство $H^{t}$ гомеоморфно $R^{m} / \mathrm{Mod}$, где $m=6 g(t)+2 n(t)-6 u \operatorname{Mod}-$ дискретная группа.

Частные случаи этой теоремы были доказаны ранее [1], [3], [4]. При $2 g(t)+n(t) \leqslant 2$ пространство $H^{t}$ имеет очень простую структуру и мы не будем обсуждать ее в этой заметке.

2. Опишем сначала топологию пространства $H^{t}$. Сопоставим морфизму $(\widetilde{P}, f, P)$ пару $(P, V)$, где $V$ - множество критических значений $f$ (т.е. $V=f(\widetilde{V})$, где $\widetilde{V}=\{\widetilde{v} \in \widetilde{P} \mid d f(\widetilde{v})=0\}$ ). Нетрудно видеть, что число точек $n=n(\widetilde{P}, f, P)$ множества $V$ и род $g=g(P)$ поверхности $P$ определяются топологическим типом морфизма $(\widetilde{P}, f, P)$. Таким образом, соответствие $(\widetilde{P}, f, P) \mapsto P \backslash V$ задает отображение $\Phi: H^{t} \rightarrow M_{g, n}$ в пространство модулей римановых поверхностей рода $g$ с $n$ проколами. Стандартная топология этого пространства хорошо известна (один из вариантов ее определения будет описан ниже). Зададим топологию на $H^{t}$, потребовав, чтобы отображение $\Phi$ было непрерывньм. (Аналогичным способом на $H^{t}$ можно ввести и комплексно-аналитическую структуру, но мы не будем использовать это в дальнейшем.)

3. Рассмотрим группу голоморфных автоморфизмов $\operatorname{Aut}(\Lambda) \cong P S L(2, \mathbb{R})$ верхней полуплоскости $\Lambda=\{z \in \mathbb{C} \mid \operatorname{In} z>0\}$. Aut $(\Lambda) \backslash$ id распадается на подмножества эллиптических $\operatorname{Aut}_{0}(\Lambda)$, параболических $\operatorname{Aut}_{1}(\Lambda)$ и гиперболических $\operatorname{Aut}_{2}(\Lambda)$ автоморфизмов, имеющих соответственно 0,1 и 2 неподвижные точки на абсолюте $\partial \Lambda=\mathbb{R} \cup \infty$.

Параболический автоморфизм с конечной неподвижной точкой $a \in \mathbb{R}$ имеет вид

$$
C(z)=\frac{(1-a \gamma) z+a^{2} \gamma}{-\gamma z+(1+a \gamma)}
$$

Назовем его положительным, если $\gamma>0$. Гиперболический автоморфизм с конечными неподвижными точками $\alpha$ и $\beta$ имеет вид

$$
C(z)=\frac{(\lambda \alpha-\beta) z+(1-\lambda) \alpha \beta}{(\lambda-1) z+(\alpha-\lambda \beta)},
$$

где $\lambda>1$. Назовем его положительным, если $\alpha<\beta$.

Для автоморфизмов $C_{1}, C_{2}$ с конечными неподвижными точками на $\mathbb{R}$ положим $C_{1}<C_{2}$, если все неподвижные точки $C_{1}$ меньше любой неподвижной точки $C_{2}$. Набор $\left\{C_{1}, C_{2}, C_{3}\right\} \subset$ $\operatorname{Aut}_{1}(\Lambda) \cup \operatorname{Aut}_{2}(\Lambda)$ назовем упорядоченныц, если $C_{1} \cdot C_{2} \cdot C_{3}=1$ и существует $D \in \operatorname{Aut}(\Lambda)$ такой, что автоморфизмы $\widetilde{C}_{i}=D C_{i} D^{-1}(i=1,2,3)$ имеют конечные неподвижные точки, положительны и $\widetilde{C}_{1}<\widetilde{C}_{2}<\widetilde{C}_{3}$. Упорядоченный набор $\left\{C_{1}, C_{2}, C_{3}\right\}$ назовем последовательныцм, если $\left\{C_{1} C_{2} C_{1}^{-1}, C_{1}, C_{3}\right\}$ также упорядоченньй набор. Набор $\left\{C_{1}, \ldots, C_{n}\right\} \subset \operatorname{Aut}_{1}(\Lambda) \cup$ $\operatorname{Aut}_{2}(\Lambda)$ назовем последовательным, если для любого $i=1, \ldots, n-1$ наборы $\left\{C_{1} \cdots C_{i-1}\right.$, $\left.C_{i}, C_{i+1} \cdots C_{n}\right\}$ - последовательные.

Работа выполнена при финансовой поддержке Российского фонда фундаментальных исследований (грант № 95-01-01122а и грант № 96-01-01104). 
4. Рассмотрим теперь свободную группу $S$ с $2 g+n-1$ образующими $\delta=\left\{a_{1}, b_{2}, \ldots, a_{g}, b_{g}\right.$, $\left.c_{1}, \ldots, c_{n-1}\right\}$. Положим

$$
c_{n}=\left(\prod_{i=1}^{\widetilde{g}}\left[a_{i} b_{i}\right] \prod_{j=1}^{n-1} c_{j}\right)^{-1} .
$$

Реализацией пары $(S, \delta)$ назовем мономорфизм $\psi: S \rightarrow \operatorname{Aut}(\Lambda)$ такой, что

$$
\left\{\psi\left(a_{1}\right), \psi\left(b_{1} a_{1}^{-1} b_{1}^{-1}\right), \ldots, \psi\left(a_{g}\right), \psi\left(b_{g} a_{g}^{-1} b_{g}^{-1}\right), \psi\left(c_{1}\right), \ldots, \psi\left(c_{n}\right)\right\}
$$

- последовательный набор, причем $\psi\left(a_{i}\right), \psi\left(b_{i}\right) \in \operatorname{Aut}_{2}(\Lambda), \psi\left(c_{j}\right) \in \operatorname{Aut}_{1}(\Lambda)(i=1, \ldots, g$, $j=1, \ldots, n)$.

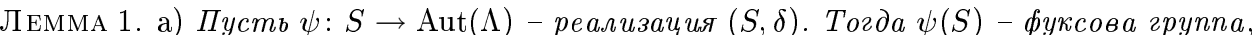
$\psi(S) \in \operatorname{Aut}_{1}(\Lambda) \cup \operatorname{Aut}_{2}(\Lambda) \cup$ id $u \Lambda / \psi(S) \in M_{g, n}$. б) Пусть $Q \in M_{g, n}$. Тогда существует реализачия $\psi$ парьц $(S, \delta)$ такая, что $\Lambda / \psi(S)=Q$.

Обозначим через $\widetilde{T}_{g, n}$ множество всех реализаций пары $(S, \delta)$. Для $\psi \in \widetilde{T}_{g, n}, s \in S, h \in$ $\operatorname{Aut}(\Lambda)$ положим $h^{*} \psi(s)=h \psi(s) h^{-1}$. Тогда $h^{*} \psi \in \widetilde{T}_{g, n}$, и при этом $\Lambda / h^{*} \psi(S)=\Lambda / \psi(S)$. Таким образом, $\operatorname{Aut}(\Lambda)$ действует на $\widetilde{T}_{g}, n$. Положим $T_{g, n}=\widetilde{T}_{g, n} / \operatorname{Aut}(\Lambda)$. Тогда соответствие $\psi \mapsto \Lambda / \psi(S)$ задает отображение $\Psi: T_{g, n} \rightarrow M_{g, n}$.

Положим $\widetilde{\operatorname{Mod}}_{g, n}=\left\{\alpha \in \operatorname{Aut}(S) \mid \widetilde{T}_{g, n} \alpha=\widetilde{T}_{g, n}\right\}$ и $\operatorname{Mod}_{g, n}=\widetilde{\operatorname{Mod}}_{g, n} / \operatorname{Int}(S)$, где $\operatorname{Int}(S)$ - множество автоморфизмов вида $s \mapsto h s h^{-1}$, где $h \in S$.

Лемма 2. а) При $2 g+n>2$ пространство $T_{g, n}$ гомеоморфно $R^{6 g+2 n-6}$. б) Соответствие $\psi \mapsto \Lambda / \psi(S)$ задает гомеоморфизм $T_{g, n} / \operatorname{Mod}_{g, n} \rightarrow M_{g, n}$, где $\operatorname{Mod}_{g, n}$ дискретно действует на $T_{g, n}$.

Доказательство лемм 1 и 2 следует из [2], [4].

5. Пусть теперь $\widetilde{S} \subset S$ - подгруппа конечного индекса группы $S$ и $\psi \in \widetilde{T}_{g, n}$. Тогда $\Lambda / \psi(\widetilde{S})$ - риманова поверхность с проколами. Вложение $\widetilde{S} \rightarrow S$ задает голоморфное накрытие $\widetilde{f}_{\psi, \widetilde{S}}: \Lambda / \psi(\widetilde{S}) \rightarrow \Lambda / \psi(S)$. Заклеив проколы точками, получим морфизм $f_{\psi, \widetilde{S}}: \widetilde{P}_{\psi, \widetilde{S}} \rightarrow P_{\psi, \widetilde{S}}$

Лемма 3. а) Эта конструкиия дает все морфизмы $(\widetilde{P}, f, P)$ с п критическими значениями на поверхностях рода $g$. б) $\left(\widetilde{P}_{h^{*} \psi, \widetilde{S}}, f_{h^{*} \psi, \widetilde{S}}, P_{h^{*} \psi, \widetilde{S}}\right)=\left(\widetilde{P}_{\psi, \widetilde{S}}, f_{\psi, \widetilde{S}}, P_{\psi, \widetilde{S}}\right)$ для любого $h \in \operatorname{Aut}(\Lambda)$. в) $\left(\widetilde{P}_{\psi_{1}, \widetilde{S}_{1}}, f_{\psi_{1}, \widetilde{S}_{1}}, P_{\psi_{1}, \widetilde{S}_{1}}\right)$ u $\left(\widetilde{P}_{\psi_{2}, \widetilde{S}_{2}}, f_{\psi_{2}, \widetilde{S}_{2}}, P_{\psi_{2}, \widetilde{S}_{2}}\right)$ имеют одинаковый топологический тип, если и только если существует автоморфизм $\alpha \in \widetilde{\operatorname{Mod}}_{g, n}$ mакой, что $\alpha\left(\widetilde{S}_{1}\right)=\widetilde{S}_{2}$.

Доказательство основано на униформизации $\Lambda \rightarrow \widetilde{P} \backslash \widetilde{V} \rightarrow P \backslash V$ и лемме 1 .

Схема доказательства теоремы. Согласно лемме 3 , существует подгруппа конечного индекса $\widetilde{S} \subset S$ такая, что соответствие $\psi \mapsto\left(\widetilde{P}_{\psi, \widetilde{S}}, f_{\psi, \widetilde{S}}, P_{\psi, \widetilde{S}}\right)$ порождает отображение $T_{g, n} / \operatorname{Mod}_{g, n}^{t} \rightarrow H^{t}$, где $\operatorname{Mod}_{g, n}^{t} \subset \operatorname{Mod}_{g, n}$. Согласно лемме $2, T_{g, n} \cong R^{6 g+2 n-6}$ и $\operatorname{Mod}_{g, n}^{t}$ дискретно действует на $T_{g, n}$.

Автор благодарит В. И. Арнольда за стимулирующие обсуждения.

\section{СПИСОК ЛИТЕРАТУРЫ}

[1] Арнольд В. И. // Функцион. анализ и его прилож. 1996. Т. 30. № 1. С. 1-19. [2] Натанзон С. М. // Труды ММО. 1978. Т. 37. С. 219-253. [3] Натанзон С. М. // Докл. АН СССР. 1986. T. 287. № 5. C. 1058-1061. [4] Natanzon S. // Amer. Math. Soc. Transl. (2). 1997. V. 180. P. $175-180$. 